\title{
ANOTHER WAY TO SAY SUBSOLUTION: THE MAXIMUM PRINCIPLE AND SUMS OF GREEN FUNCTIONS
}

\author{
R. S. LAUGESEN and N. A. WATSON*
}

\begin{abstract}
Consider an elliptic second order differential operator $L$ with no zeroth order term (for example the Laplacian $L=-\Delta$ ). If $L u \leq 0$ in a domain $U$, then of course $u$ satisfies the maximum principle on every subdomain $V \subset U$.

We prove a converse, namely that $L u \leq 0$ on $U$ if on every subdomain $V$, the maximum principle is satisfied by $u+v$ whenever $v$ is a finite linear combination (with positive coefficients) of Green functions with poles outside $\bar{V}$.

This extends a result of Crandall and Zhang for the Laplacian.

We also treat the heat equation, improving Crandall and Wang's recent result. The general parabolic case remains open.
\end{abstract}

\section{Introduction}

We will extend the following "maximum principle characterization" of subharmonic functions, given by Crandall and Zhang in their paper Another way to say harmonic [5], to a wide class of second order elliptic operators with no zeroth order terms.

Write $K(r)=1 / r^{n-2}$ for the radially symmetric fundamental solution of Laplace's equation, when $n \neq 2$, with $K(r)=\log (1 / r)$ when $n=2$. Also write $K(x)=K(|x|)$ when $x \in \mathrm{R}^{n}$.

Theorem 1.1 ([5], Theorem 2.4). Let $u: U \rightarrow \mathrm{R}$ be upper semicontinuous on an open set $U \subset \mathbf{R}^{n}, n \geq 1$. Suppose $u+v$ satisfies the maximum principle on $V$ whenever $V$ is a bounded open set with $\bar{V} \subset U$ and $v$ has the form

$$
v(x)=\sum_{m=1}^{n} a_{m} K\left(x-\xi_{m}\right)
$$

for some positive real numbers $a_{1}, \ldots, a_{n}$ and some points $\xi_{1}, \ldots, \xi_{n} \in \mathrm{R}^{n} \backslash \bar{V}$. (In other words $\max _{\bar{V}}(u+v)=\max _{\partial V}(u+v)$ for all such $v$ and $V$.)

\footnotetext{
* Laugesen was partially supported by National Science Foundation Award DMS-0140481 and a Maclaurin Fellowship from the New Zealand Institute of Mathematics and its Applications.

Received July 30, 2004.
} 


\section{Then $u$ is subharmonic.}

Note that $v$ is a positive linear combination of $n$ fundamental solutions with distinct singularities outside $\bar{V}$. The number " $n$ " of terms here is minimal, as Crandall and Zhang observed.

The converse to Theorem 1.1 is trivial: if $u$ is subharmonic on $V$ then so is $u+v$, because $v$ is harmonic on $V$, and thus $u+v$ does indeed satisfy the maximum principle on $V$.

Our main goal is to find a similar characterization of subsolutions in the elliptic case. This we do in Theorem 2.6 of Section 2. Our Theorem 2.6 is worse than Crandall and Zhang's Theorem 1.1 because our linear combinations $v$ employ arbitrarily many fundamental solutions, rather than just $n$ of them. Perhaps this is unavoidable, because there is no explicit formula for the fundamental solution in the general elliptic case and hence there is much less information to work with. Instead we proceed by a potential theoretic line of proof that is less explicit and more flexible.

Theorem 2.6 is in other ways better than Theorem 1.1, because it uses Green functions (of which fundamental solutions on all of space are just a special case, when $n \geq 3$ ).

Our second goal, in Section 3, is to strengthen Theorem 1.1 for subharmonic functions - we consider $u-v$ as well as $u+v$, and we localize the singularities.

In Section 4 we strengthen Crandall and Wang's analogous characterization [4] of subsolutions of the heat equation, using linear combinations of just $n$ Gaussian fundamental solutions in $\mathrm{R}^{n+1}$ (whereas [4] needed $n+1$ Gaussians). A natural question is whether subsolutions of more general parabolic equations can be characterized similarly, in terms of the maximum principle and Green functions. Technical difficulties have so far prevented us from dealing with this parabolic case, and so the question remains open.

Incidentally, this line of research began when subsolutions of the $\infty$-Laplace equation were characterized by Crandall, Evans and Gariepy [3] in terms of the maximum principle and cone functions:

if $u+v$ satisfies the maximum principle on $V$ whenever $V$ is a bounded open set with $\bar{V} \subset U$ and $v(x)=-a|x-\xi|$ for some $a>0$ and $\xi \in \mathrm{R}^{n} \backslash \bar{V}$, then $u$ is $\infty$-subharmonic, meaning $-D u \cdot\left(D^{2} u\right) D u \leq 0$.

Here $D u$ is the gradient vector of $u$ and $D^{2} u$ is the Hessian. For a strikingly simple proof of this fact, see [5, Section 2]. It remains an open problem to similarly characterize subsolutions of the $p$-Laplace equation, for $p \neq 2, \infty$. Partial results are in [5, Section 3]. For the $\infty$-heat equation, see [4, Section 3]. The $p$-heat equation seems not to have been investigated in this regard, for $p \neq 2, \infty$. 


\section{Subsolutions of elliptic equations}

Our characterization of subsolutions comes in Theorem 2.6 below. But first we establish some notation and lemmas.

Note. We will not consider dimension $n=1$ because the potential theory there is different (for example the Green function is finite on the diagonal) and thus would require special treatment.

\subsection{Definitions}

Let $\Lambda$ be an open subset of $\mathrm{R}^{n}, n \geq 2$. We consider in $\Lambda$ the elliptic operator $L$ with real coefficients and no zeroth order term:

$$
L u=-\sum_{i, j=1}^{n} a_{i j} D_{i} D_{j} u+\sum_{i=1}^{n} b_{i} D_{i} u,
$$

where $a_{i j} \in C^{2+\delta}(\Lambda), b_{i} \in C^{1+\delta}(\Lambda)$, and $a_{i j}=a_{j i}$. The ellipticity condition is

$$
\sum_{i, j=1}^{n} a_{i j}(x) \xi_{i} \xi_{j} \geq \theta|\xi|^{2}
$$

for all $x \in \Lambda$ and $\xi \in \mathrm{R}^{n}$. These assumptions on the coefficients are sufficient for the potential theoretic arguments we use later on.

We shall give a maximum principle characterization of the subsolutions associated with the operator $L$. The known results that we shall use appeared in [2] and [6]. We follow the potential theoretic terminology of those references.

Definition 2.1. A function $u: U \rightarrow[-\infty,+\infty)$ defined on an open subset $U$ of $\Lambda$ is called $L$-hypoharmonic if it satisfies the following conditions:

(i) $u$ is upper semicontinuous;

(ii) for each $L$-regular open set $D \Subset U$ we have the inequality

$$
u(x) \leq \int u d \rho_{x}^{D} \quad \text { for every } \quad x \in D,
$$

where $\rho_{x}^{D}$ denotes the $L$-harmonic measure relative to $D$ and $x$ (see [2, p. 62]).

An $L$-hypoharmonic function on $U$ is called $L$-subharmonic if it is finite at some point in each component of $U$. A function $u$ is called $L$-superharmonic if $-u$ is $L$-subharmonic.

The point of $L$-hypoharmonicity is that on every $L$-regular subdomain $D$, the function $u$ is majorized by the $L$-harmonic function equalling $u$ on $\partial D$ (if there is one). 
REMARK. Littman [8] studied weakly L-subharmonic functions, which are locally integrable functions $u$ on $U$ satisfying

$$
\int u(x) L^{*} v(x) d x \leq 0
$$

whenever $v \geq 0$ and $v \in C_{c}^{2}(U)$, where $L^{*}$ is the formal adjoint of $L$. Note that every weakly $L$-subharmonic function is equal a.e. to an $L$-subharmonic function, and conversely, as demonstrated in [12] (with the additional, but superfluous, hypothesis that $L$ is self-adjoint).

We shall use the local criterion for $L$-hypoharmonicity given in [2, p. 72]. Thus $u$ is $L$-hypoharmonic if $u: U \rightarrow[-\infty,+\infty)$ is upper semicontinuous and the inequality in (ii) holds for each $D$ in some base for the Euclidean topology on $U$ that consists entirely of $L$-regular open sets.

We employ a particular type of base for the topology of $U$. Fix a bounded convex domain $B$ that contains the origin, fix $\epsilon>0$, and put

$$
\mathrm{B}(U)=\mathrm{B}_{B, \epsilon}(U)=\left\{x+\delta B: x \in \mathrm{R}^{n}, 0<\delta<\epsilon, \overline{x+\delta B} \subset U\right\} .
$$

Then $\mathrm{B}(U)$ is a base which consists entirely of $L$-regular sets, because every convex domain is $\Delta$-regular and regularity does not vary with the coefficients of the operator, under our conditions on the $a_{i j}$ and $b_{i}$ (see [6] or [11]).

Let $V$ be an open set, and let $u$ be upper semicontinuous and upper finite on $\bar{V}$. We say that $u$ satisfies the maximum principle on $V$ if

$$
\max _{\bar{V}} u=\max _{\partial V} u
$$

Note that $L$-hypoharmonic functions satisfy the maximum principle on every $V \Subset U$, by [2, p. 72].

\subsection{Preparatory lemmas}

The first lemma below indicates the direction in which we shall go.

Given an open subset $V$ of $\Lambda$, we denote by $H_{L}(V)$ the class of all $L$ harmonic functions on $V$, such a function being a classical solution of $L u=0$. We also denote by $C(\bar{V})$ the class of all continuous functions on $\bar{V}$. If $V$ is a regular set and $f$ is a continuous real-valued function on $\partial V$, then we denote by $H_{f}^{V}$ the function on $\bar{V}$ that belongs to $C(\bar{V}) \cap H_{L}(V)$ and coincides with $f$ on $\partial V$; in other words the solution of the Dirichlet problem on $V$ with boundary data $f$. By definition of $L$-harmonic measure [2, p. 62], one has $H_{f}^{V}(x)=\int f d \rho_{x}^{V}$ for $x \in V$.

Lemma 2.2. Let $u: U \rightarrow[-\infty,+\infty)$ be upper semicontinuous on an open subset $U$ of $\Lambda$. Suppose either 
(a) $u+v$ satisfies the maximum principle on $V$ whenever $V \in \mathrm{B}(U)$ and $v \in C(\bar{V}) \cap H_{L}(V)$; or else

(b) $u-v$ satisfies the maximum principle on $V$ whenever $V \in \mathrm{B}(U)$ and $v \in C(\bar{V}) \cap H_{L}(V)$.

Then $u$ is L-hypoharmonic on $U$.

Proof of Lemma 2.2. We need only prove part (a), since $-v \in C(\bar{V}) \cap$ $H_{L}(V)$ if and only if $v \in C(\bar{V}) \cap H_{L}(V)$.

Fix $V \in \mathrm{B}(U)$. Since the restriction of $u$ to $\partial V$ is upper semicontinuous and upper finite, there is a decreasing sequence of real-valued continuous functions $\left\{f_{k}\right\}$ on $\partial V$ such that $\lim _{k \rightarrow \infty} f_{k}=u$. Since $V$ is regular, for each $k$ we can define $v_{k} \in C(\bar{V}) \cap H_{L}(V)$ by putting $v_{k}=H_{f_{k}}^{V}$. Then $v_{k} \geq u$ on $\partial V$, and $u-v_{k}$ is upper semicontinuous and upper finite on $\bar{V}$, so that the maximum principle hypothesis implies $u \leq v_{k}$ on $V$. Since $\left\{f_{k}\right\}$ is decreasing, so is $\left\{v_{k}\right\}$, and hence for all $x \in V$ we have

$$
u(x) \leq \lim _{k \rightarrow \infty} v_{k}(x)=\lim _{k \rightarrow \infty} \int f_{k} d \rho_{x}^{V}=\int u d \rho_{x}^{V} .
$$

Since this holds for all $V \in \mathrm{B}(U)$, the local criterion for $L$-hypoharmonicity shows $u$ is $L$-hypoharmonic.

REMARK. The function $u$ by itself might satisfy the maximum principle on every $V$ without $u$ being $L$-hypoharmonic, because subsolutions of any operator having the same form as $L$ will satisfy the maximum principle. For example, the harmonic function $u\left(x_{1}, x_{2}\right)=x_{1}^{2}-x_{2}^{2}$ satisfies the maximum principle on every subdomain of $\mathrm{R}^{2}$, but is not $L$-hypoharmonic for $L=$ $-\left(D_{1}^{2}+2 D_{2}^{2}\right)$ because $L u=2>0$.

The main result of this section, Theorem 2.6, will confine the test functions $v$ required in Lemma 2.2 to a smaller class. For its proof we require two further lemmas, both of which extend known results for the harmonic case $L=-\Delta$.

The first lemma is a criterion for $L$-superharmonicity that extends [1, Corollary 3.2.4].

LEMMA 2.3. Let $u$ be an L-superharmonic function on an open set $U$ and let $h$ be an $L$-superharmonic function on an open subset $V$ of $U$. If

$$
u(y) \leq \liminf _{x \rightarrow y, x \in V} h(x)
$$

for all $y \in U \cap \partial V$, then the function

$$
w= \begin{cases}\min (h, u) & \text { on } V, \\ u & \text { on } U \backslash V,\end{cases}
$$


is L-superharmonic on $U$.

Proof of Lemma 2.3. Clearly $w>-\infty$ on $U$, because $u>-\infty$ and $h>-\infty$. Also, $w$ is finite at some point in each component of $U$, since $w \leq u$. Condition (2) implies that $w$ is lower semicontinuous at every point of $U \cap \partial V$, and therefore on $U$. To complete the proof, we use the local criterion for $L$ superharmonicity. Clearly the criterion is satisfied at every point of $U \backslash \partial V$. Let $\xi_{0} \in U \cap \partial V$, and let $D$ be a regular open set such that $\xi_{0} \in D$ and $\bar{D} \subset U$. Then

$$
w\left(\xi_{0}\right)=u\left(\xi_{0}\right) \geq \int u d \rho_{\xi_{0}}^{D} \geq \int w d \rho_{\xi_{0}}^{D},
$$

so that the local criterion is satisfied at $\xi_{0}$. Hence $w$ is $L$-superharmonic on $U$.

Lemma 2.3 is used in the proof of the Extension Lemma below, which extends the harmonic case $L=-\Delta$ in [1, p. 192], by following a broadly similar proof. For this Extension Lemma we require the classical notion of a Green function for the Dirichlet problem relative to the operator $L$.

Definition 2.4. A classical L-Green function on a subdomain $\Gamma$ of $\Lambda$ is a continuous, extended-real-valued function $G: \Gamma \times \Gamma \rightarrow(0, \infty]$ with the following properties for each $\xi \in \Gamma$ :

(i) $L G(\cdot, \xi)=0$ (in the classical sense) on $\Gamma \backslash\{\xi\}$;

(ii) $G(\xi, \xi)=\infty$;

(iii) $G(\cdot, \xi)$ can be continuously extended to 0 on $\partial \Gamma$ (including the point at infinity if $\Gamma$ is unbounded).

Traditionally, a Green function's singularity must also be of a prescribed type (see [9, p. 20], for example), but we don't need that property below.

If $\Gamma$ is a regular set and $\bar{\Gamma}$ is a compact subset of $\Lambda$, then it follows from [9, p. 64] that $\Gamma$ possesses a classical $L$-Green function. If $L=-\Delta$ and $n \geq 3$, then $\Lambda=\mathrm{R}^{n}$ itself possess a classical Green function, namely $G(x, \xi)=$ $|x-\xi|^{2-n}$.

In the proof of the Extension Lemma, it will be convenient to use level sets of the $L$-Green function $G\left(\cdot, \xi_{0}\right)$ with pole at a point $\xi_{0}$. For any $\xi_{0} \in \Gamma$ and $r>0$ such that $K(r)>0$, we put

$$
B_{\Gamma}\left(\xi_{0}, r\right)=\left\{x \in \Gamma: G\left(x, \xi_{0}\right)>K(r)\right\},
$$

and call $B_{\Gamma}\left(\xi_{0}, r\right)$ the $L$-Green ball with centre $\xi_{0}$ and radius $r$. Note that if $L=-\Delta$ and $\Lambda=\mathrm{R}^{n}$ with Green function as above, then $B_{\Gamma}\left(\xi_{0}, r\right)$ is the Euclidean open ball with centre $\xi_{0}$ and radius $r$.

In general, $B_{\Gamma}\left(\xi_{0}, r\right)$ is an open set with closure in $\Gamma$. It is also connected, because if it had a component $U$ that did not contain $\xi_{0}$, we would have 
$G\left(\cdot, \xi_{0}\right)=K(r)$ on $\partial U$ and $L G\left(\cdot, \xi_{0}\right)=0$ throughout $U$, which would yield $G\left(\cdot, \xi_{0}\right)=K(r)$ on $U$, contradicting the definition of $B_{\Gamma}\left(\xi_{0}, r\right)$.

Further, $B_{\Gamma}\left(\xi_{0}, r\right)$ expands to fill $\Gamma$ as $r \rightarrow \infty$ if $n \geq 3$, or as $r \rightarrow 1$ if $n=2$, since $K(r) \rightarrow 0$.

Lemma 2.5 (Extension Lemma). Let $\Gamma$ be a subdomain of $\Lambda$ that has a classical L-Green function $G$. Let $C$ be a convex compact subset of $\Gamma$, and let $v$ be an L-superharmonic function on an open set containing $C$.

Then given any point $\xi_{0} \in \Gamma$, there is an L-superharmonic function $\bar{v}$ on $\Gamma$ such that $\bar{v}=v$ on a neighbourhood of $C$, and $\bar{v}=\alpha+\beta G\left(\cdot, \xi_{0}\right)$ on $\Gamma \backslash B_{\Gamma}\left(\xi_{0}, \rho\right)$ for some real constants $\alpha, \beta, \rho$ with $\beta, \rho>0$. Furthermore, $\bar{v}-\alpha>0$ on $\Gamma$.

Proof of Lemma 2.5. Let $U$ be a bounded open set containing $C$, such that $v$ is $L$-superharmonic and lower bounded on $U$. By adding a constant if necessary, we can assume that $v>0$ on $U$. Choose $R>0$ such that $\bar{U} \subset B_{\Gamma}\left(\xi_{0}, R\right)$.

Our first aim is to show that there is an $L$-superharmonic function $w$ on $B_{\Gamma}\left(\xi_{0}, R+r\right)$ such that $w=v$ on a neighbourhood of $C$, where $r=1$ if $n \geq 3$ and $r=(1-R) / 2$ if $n=2$. Let $D$ be a convex domain such that $C \subset D \Subset U$. Put $u=\widehat{\mathrm{R}}_{v}^{D}$, the regularized reduced function (or balayage) of $v$ relative to $D$ in $U$ (see [2, p. 80]). Then $u$ is $L$-superharmonic on $U$ by [2, p. 77], and $L$-harmonic on $U \backslash \bar{D}$, by [2, p. 84], with $0 \leq u \leq v$ on $U$ and $u=v$ on $D$ by [2, p. 81]. (In other words, $u$ solves the obstacle problem with $v$ as the obstacle on $D$.)

The next step in the construction of $w$ is to let $E$ be a convex compact set such that $\bar{D} \subset E^{o}$ and $E \subset U$. Put $A=B_{\Gamma}\left(\xi_{0}, R+r\right) \backslash E$, so that $A$ is a kind of "annular" region. The convexity of $E$ ensures every point of $\partial E$ is a regular point for the $L$-Dirichlet problem on $A$, because every such point is regular for Laplace's equation and regularity does not vary with the coefficients of the operator, as remarked earlier. (Incidentally, here we use exterior regularity of the convex set. Earlier we used interior regularity.) Furthermore, because $G\left(\cdot, \xi_{0}\right)-K(R+r)$ is a positive $L$-superharmonic function on $A$ that tends to zero at every point of $\partial B_{\Gamma}\left(\xi_{0}, R+r\right)$, every such boundary point is also regular for the $L$-Dirichlet problem on $A$, by the "barrier" criterion in [2, p. 118]. Hence $A$ is a regular set.

Next put $g_{1}=u$ on $\partial E$ and $g_{1}=0$ on $\partial B_{\Gamma}\left(\xi_{0}, R+r\right)$, and put $g_{2}=0$ on $\partial E$ and $g_{2}=1$ on $\partial B_{\Gamma}\left(\xi_{0}, R+r\right)$. Let $h_{k}=H_{g_{1}}^{A}-k H_{g_{2}}^{A}$ for each $k \in \mathrm{N}$. Note that $u$ is continuous on $\partial E$ because $u$ is $L$-harmonic on $U \backslash \bar{D}$. Since $B_{\Gamma}\left(\xi_{0}, R+r\right)$ is connected and $E$ is convex, $A$ is connected. Therefore $H_{g_{2}}^{A}>0$ on $A$, so that $\left\{h_{k}\right\}$ decreases to $-\infty$ in $A$ as $k \rightarrow \infty$. Choose $m$ such that $h_{m} \leq 0$ on $\partial U$. Since $A$ is regular, $h_{m}(x) \rightarrow u(y)$ as $x \rightarrow y$, for every $y \in \partial E$. Since we 
also have $h_{m} \leq 0$ on $\partial U$ and $u \geq 0$ on $U$, the minimum principle ensures that $h_{m} \leq u$ on $U \backslash E$.

Now define $w=u$ on $E, w=h_{m}$ on A. An application of Lemma 2.3 (with $V=U \backslash E$ and $h=h_{m}$ ) shows that $w$ is $L$-superharmonic on $U$, and hence on $B_{\Gamma}\left(\xi_{0}, R+r\right)$. Clearly $w$ is $L$-harmonic on $A$, with $w=u=v$ on $D$, and $w$ tends to $-m$ on $\partial B_{\Gamma}\left(\xi_{0}, R+r\right)$.

Our second aim in the proof is to extend $w$ from $B_{\Gamma}\left(\xi_{0}, R+r\right)$ to all of $\Gamma$, thereby obtaining $\bar{v}$. Since $w$ is $L$-harmonic on $A$, it is continuous on $\partial B_{\Gamma}\left(\xi_{0}, R\right)$ and so it has a maximum value $M(R)$ there. We choose $\beta>0$ such that

$$
\beta(K(R)-K(R+r)) \geq M(R)+m,
$$

so that

$$
\beta K(R)-M(R) \geq \beta K(R+r)+m .
$$

Then we choose $\alpha \in \mathrm{R}$ such that

$$
\beta K(R)-M(R) \geq-\alpha \geq \beta K(R+r)+m,
$$

so that

$$
\alpha+\beta K(R) \geq M(R) \quad \text { and } \quad \alpha+\beta K(R+r) \leq-m .
$$

Thus

$$
\begin{array}{ll}
\alpha+\beta G\left(\cdot, \xi_{0}\right) \geq M(R) & \text { on } \quad \partial B_{\Gamma}\left(\xi_{0}, R\right), \\
\alpha+\beta G\left(\cdot, \xi_{0}\right) \leq-m & \text { on } \quad \partial B_{\Gamma}\left(\xi_{0}, R+r\right) .
\end{array}
$$

Now we define

$$
\bar{v}= \begin{cases}w & \text { on } \overline{B_{\Gamma}\left(\xi_{0}, R\right)}, \\ \min \left(w, \alpha+\beta G\left(\cdot, \xi_{0}\right)\right) & \text { on } B_{\Gamma}\left(\xi_{0}, R+r\right) \backslash \overline{B_{\Gamma}\left(\xi_{0}, R\right)}, \\ \alpha+\beta G\left(\cdot, \xi_{0}\right) & \text { on } \Gamma \backslash B_{\Gamma}\left(\xi_{0}, R+r\right),\end{cases}
$$

and note that $\bar{v}=w=v$ on $D$. An application of Lemma 2.3 (with $U=$ $B_{\Gamma}\left(\xi_{0}, R+r\right), V=B_{\Gamma}\left(\xi_{0}, R+r\right) \backslash \overline{B_{\Gamma}\left(\xi_{0}, R\right)}, u=w$ and $\left.h=\alpha+\beta G\left(\cdot, \xi_{0}\right)\right)$ shows that $\bar{v}$ is $L$-superharmonic on $B_{\Gamma}\left(\xi_{0}, R+r\right)$. Another application of Lemma 2.3 (with $U=\Gamma \backslash B_{\Gamma}\left(\xi_{0}, R\right), V=B_{\Gamma}\left(\xi_{0}, R+r\right) \backslash \overline{B_{\Gamma}\left(\xi_{0}, R\right)}$, $u=\alpha+\beta G\left(\cdot, \xi_{0}\right)$ and $\left.h=w\right)$ shows that $\bar{v}$ is also $L$-superharmonic on $\Gamma \backslash \overline{B_{\Gamma}\left(\xi_{0}, R\right)}$, and hence $\bar{v}$ is $L$-superharmonic on $\Gamma$. Finally, since $\bar{v}-\alpha=$ $\beta G\left(\cdot, \xi_{0}\right)>0$ outside a compact subset of $\Gamma$, we conclude $\bar{v}-\alpha>0$ on $\Gamma$. 


\subsection{The characterization of subsolutions}

We now come to our main result, Theorem 2.6, in which Lemma 2.2 is improved by replacing the arbitrary $v \in C(\bar{V}) \cap H_{L}(V)$ with a finite linear combination (having positive coefficients) of $L$-Green functions with poles outside $\bar{V}$.

TheOREM 2.6. Let $u: U \rightarrow[-\infty,+\infty)$ be upper semicontinuous on an open subset $U$ of $\Lambda$. Suppose $U$ is contained in a domain $\Gamma \subset \Lambda$ which has a classical L-Green function $G$. Assume either

(a) $u+v$ satisfies the maximum principle on $V$ whenever $V \in \mathrm{B}(U)$ and $v$ has the form

$$
v=\sum_{m=1}^{M} a_{m} G\left(\cdot, \xi_{m}\right)
$$

for some $M \in \mathrm{N}$, some positive real numbers $a_{1}, \ldots, a_{M}$, and some distinct points $\xi_{1}, \ldots, \xi_{M} \in U \backslash \bar{V}$; or else

(b) $u-v$ satisfies the maximum principle on $V$ whenever $V \in \mathrm{B}(U)$ and $v$ has the form (3).

Then $u$ is L-hypoharmonic on $U$.

For example if $L=-\Delta$ is the Laplacian then one could take the Green function to be the fundamental solution $K(x-\xi)$ on all of space, provided $n \geq 3$. (When $n=2$, the fundamental solution fails to be a Green function because it is negative near infinity.) Thus Theorem 2.6 implies the CrandallZhang result Theorem 1.1 on subharmonic functions, when $n \geq 3$, except using linear combinations $v$ that have arbitrarily many terms. See Section 3 for more on this subharmonic case.

Proof of Theorem 2.6. Part (a). In view of Lemma 2.2(a), it suffices to prove that $u+v$ satisfies the maximum principle on $V$ whenever $V \in \mathrm{B}(U)$ and $v \in C(\bar{V}) \cap H_{L}(V)$. We prove the contrapositive. Suppose that we can find $V \in \mathrm{B}(U)$ and $v \in C(\bar{V}) \cap H_{L}(V)$ such that

$$
\max _{\bar{V}}(u+v)>\max _{\partial V}(u+v) .
$$

We will show the same inequality holds on a slightly smaller set $V_{1} \in \mathrm{B}(U)$.

Define $w=u+v$ on $\bar{V}$. Then there is $y_{0} \in V$ such that $w\left(y_{0}\right)>w(y)$ for all $y \in \partial V$. Since $w$ is upper semicontinuous and $\partial V$ is compact, $w\left(y_{0}\right)>w(y)$ for all $y$ in some neighborhood of $\partial V$. Hence we can find $V_{1} \in \mathrm{B}(U)$ such that $y_{0} \in V_{1} \Subset V$ and $w\left(y_{0}\right)>w(y)$ for all $y \in \partial V_{1}$ (for instance one could write $V=x+\delta B$ as in the definition (1) and then put $V_{1}=x+\delta_{1} B$ where 
$\delta_{1} \in(0, \delta)$ is sufficiently close to $\delta$, using here that $B$ is convex and contains the origin). Thus

$$
\max _{\overline{V_{1}}}(u+v)>\max _{\partial V_{1}}(u+v),
$$

and $v$ is defined on an open set that contains $\overline{V_{1}}$.

We now choose $\varepsilon$ such that

$$
0<\varepsilon<(u+v)\left(y_{0}\right)-\max _{\partial V_{1}}(u+v) .
$$

By the Extension Lemma (with $C=\overline{V_{1}}$ ), there is an $L$-superharmonic function $\bar{v}$ on $\Gamma$ such that $\bar{v}=v$ on some bounded open set $W$ with $\overline{V_{1}} \subset$ $W \subset V$, and $\bar{v}-\alpha>0$ on $\Gamma$ for some $\alpha \in \mathrm{R}$. We now take the regularized reduced function (or balayage) of $\bar{v}-\alpha$ relative to $W$ in $\Gamma$ : let $s=\widehat{\mathrm{R}}_{\bar{v}-\alpha}^{W}$ so that $s=\bar{v}-\alpha=v-\alpha$ on $W$ and $s$ is $L$-harmonic on $\Gamma \backslash \bar{W}$ (cf. the proof of the Extension Lemma). Hence $s$ is $L$-harmonic on $\Gamma \backslash \partial W$. Since $W$ is relatively compact, $s$ is an $L$-potential on $\Gamma$ by [2, p. 94].

The $L$-potentials on $\Gamma$ with point support are proportional, by [6, p. 563], so that there is a nonnegative Radon measure $\mu$ on $\Gamma$ such that

$$
s(x)=\int_{\Gamma} G(x, \xi) d \mu(\xi)
$$

for all $x \in \Gamma$, by [6, p. 481]. Since $s$ is $L$-harmonic on $\Gamma \backslash \partial W$, we know $\mu$ is supported on $\partial W$ in view of [6, pp. 464-5]. For each fixed $\xi \in \Gamma$ the function $G(\cdot, \xi)$ is an $L$-potential with point support, and hence $G$ is continuous off the diagonal by [6, p. 480]. Also $G$ is finite off the diagonal, by definition. It follows that $G$ is uniformly continuous on $\overline{V_{1}} \times \partial W$, so that we can find $\eta>0$ such that

$$
|G(x, \zeta)-G(x, \xi)|<\frac{\varepsilon}{2 \mu(\partial W)}
$$

whenever $x \in \overline{V_{1}}$ and $\xi, \zeta \in \partial W$ with $|\xi-\zeta|<\eta$. (Note $\mu(\partial W)>0$ because otherwise $s \equiv 0$, contradicting that $s=\bar{v}-\alpha>0$ on $W$.) We now partition $\partial W$ into finitely many disjoint subsets $E_{1}, \ldots, E_{M}$, such that $\mu\left(E_{m}\right)>0$ and $\operatorname{diam}\left(E_{m}\right)<\eta$ for all $m=1, \ldots, M$. We choose points $\xi_{1}, \ldots, \xi_{M}$ such that $\xi_{m} \in E_{m}$ for all $m$, and put

$$
v_{1}(x)=\sum_{m=1}^{M} \mu\left(E_{m}\right) G\left(x, \xi_{m}\right)
$$

for all $x \in \Gamma$. Then $v_{1}$ has the form (3) relative to $V_{1}$, because $\xi_{1}, \ldots, \xi_{M}$ lie outside $\overline{V_{1}}$. Clearly $v_{1}$ is a Riemann sum approximation to $s$, and indeed for 
all $x \in \overline{V_{1}}$ we have

$$
\begin{aligned}
\left|s(x)-v_{1}(x)\right| & =\left|\sum_{m=1}^{M} \int_{E_{m}}\left(G(x, \xi)-G\left(x, \xi_{m}\right)\right) d \mu(\xi)\right| \\
& \leq \sum_{m=1}^{M} \int_{E_{m}}\left|G(x, \xi)-G\left(x, \xi_{m}\right)\right| d \mu(\xi) \\
& <\sum_{m=1}^{M} \frac{\varepsilon}{2 \mu(\partial W)} \mu\left(E_{m}\right) \\
& =\frac{\varepsilon}{2}
\end{aligned}
$$

Therefore

$$
\begin{aligned}
\max _{\partial V_{1}}\left(u+v_{1}\right) & \leq \max _{\partial V_{1}}(u+s)+\frac{\varepsilon}{2} & & \text { by (4) } \\
& =\max _{\partial V_{1}}(u+v-\alpha)+\frac{\varepsilon}{2} & & \text { since } s=v-\alpha \text { on } W \\
& <(u+v)\left(y_{0}\right)-\alpha-\frac{\varepsilon}{2} & & \text { by choice of } \varepsilon \\
& =(u+s)\left(y_{0}\right)-\frac{\varepsilon}{2} & & \text { since } s=v-\alpha \text { on } W \\
& <\left(u+v_{1}\right)\left(y_{0}\right) & & \text { by (4). }
\end{aligned}
$$

Thus we have found $V_{1} \in \mathrm{B}(U)$ and $v_{1}$ of the form (3) such that $u+v_{1}$ does not satisfy the maximum principle on $V_{1}$. This proves the contrapositive and establishes the theorem.

Part (b). Simply make the obvious changes to the proof of part (a), using $u-v$ instead of $u+v$, and using Lemma 2.2(b) at the beginning instead of Lemma 2.2(a).

\section{Subharmonic functions}

\subsection{The characterization}

The characterization of subsolutions in Theorem 2.6 takes a particularly attractive form for the Laplacian $L=-\Delta$, because one can fix the number of terms in the linear combinations to be $M=n$ provided one employs the fundamental solution instead of a Green function. This is the content of Theorem 1.1, due to Crandall and Zhang.

We make two improvements to that result, in Theorem 3.1 below. First, we handle $u-v$ as well as $u+v$ in the hypotheses - this does require proof. 
Second, we localize the singularities $\xi_{m}$ to belong to $U$ (whereas Crandall and Zhang considered all $\xi_{m}$ in $\mathrm{R}^{n}$, in their hypotheses). It is natural to seek localized singularities because subharmonicity is itself a local property. Note that the singularities are already localized to $U$ in our elliptic characterization theorem, Theorem 2.6.

We also simplify Crandall and Zhang's proof: see the remarks after the theorem below.

Recall the fundamental solution $K(x)=K(|x|)$ of the Laplacian from Section 1.

THEOREM 3.1. Let $u: U \rightarrow \mathrm{R}$ where $U$ is an open set in $\mathrm{R}^{n}, n \geq 1$, with neighborhood base $\mathscr{B}(U)$. Suppose $u \in C^{2}(U)$. Assume either

(a) $u+v$ satisfies the maximum principle on $V$ whenever $V \in \mathscr{B}(U)$ and $v$ has the form

$$
v=\sum_{m=1}^{n} a_{m} K\left(\cdot-\xi_{m}\right)
$$

for some positive real numbers $a_{1}, \ldots, a_{n}$, and some distinct points $\xi_{1}, \ldots, \xi_{n} \in U \backslash \bar{V}$; or else

(b) $u-v$ satisfies the maximum principle on $V$ whenever $V \in \mathscr{B}(U)$ and $v$ has the form (5).

Then $u$ is subharmonic in $U$.

COROLlary 3.2. Theorem 3.1 still holds if the hypotheses $u: U \rightarrow \mathrm{R}$ and $u \in C^{2}(U)$ are weakened to $u: U \rightarrow[-\infty, \infty)$ being upper semicontinuous with $u \neq \equiv-\infty$.

Remarks on Theorem 3.1. 1. The neighborhood base $\mathscr{B}(U)$ here is arbitrary, unlike in Theorem 2.6 where each $V \in \mathrm{B}(U)$ must be convex.

2. In one dimension $(n=1)$ the fundamental solution is $K(x)=-|x|$. Then $v(x)=a_{1} K\left(x-\xi_{1}\right)$ is linear on the interval $V$, and can be either increasing or decreasing depending on whether the singularity $\xi_{1}$ is to the right or left of $V$. Thus in one dimension, Theorem 3.1 says $u$ is convex on an interval $U$ if for every subinterval $V \subset U$ and every linear function $v$ on $V$, the sum $u+v$ attains its maximum at an endpoint of $V$. This statement is easily proved by the contrapositive.

3. To prove the theorem and corollary below, we follow Crandall and Zhang's method. But we introduce a new parameter $\lambda$ to allow localization of the singularities. And we perturb the standard basis $\left\{e_{1}, \ldots, e_{n}\right\}$ for $\mathrm{R}^{n}$ to the basis $\left\{q_{1}, \ldots, q_{n}\right\}$, in estimate (9) in the proof, thereby eliminating the tricky "non-generic" case of Crandall and Zhang. 
A further simplification is the removal of the case $D u\left(x_{0}\right)=0$, which required separate treatment in [5, p. 251, 253]. In Theorem 3.1 we remove this case by a simple perturbation. In Corollary 3.2 we avoid it by employing a weaker-than-usual notion of viscosity subsolution, namely a definition that has the restriction $D \phi\left(x_{0}\right) \neq 0$ imposed on the test functions [7, Definition 2.5].

4. Crandall and Zhang also proved a version of Theorem 1.1 in which the $n$-th term in the linear combination $v(x)$ is changed from $a_{n} K\left(x-\xi_{n}\right)$ to $x \cdot \xi_{n}$ and the coefficients are $a_{m}=1$ for $m=1, \ldots, n-1$. The proof is similar but easier, and our proof below can be adapted to this version also.

Proof of Theorem 3.1. Part (a). To establish the contrapositive we assume $u$ is not subharmonic, meaning $\Delta u\left(x_{0}\right)<0$ at some point $x_{0} \in U$. We may suppose the gradient vector $D u\left(x_{0}\right) \neq 0$, just by shifting $x_{0}$ slightly if necessary; this is possible since $D u \equiv 0$ in a neighborhood of $x_{0}$ would imply $\Delta u\left(x_{0}\right)=0$.

By a translation we may further suppose $x_{0}=0$, so that we have

$$
\Delta u(0)<0 \quad \text { and } \quad D u(0) \neq 0 .
$$

We will choose $a_{m}>0$ and $\xi_{m} \in U$ distinct in such a way that

$$
v(x)=\sum_{m=1}^{n} a_{m} K\left(x-\xi_{m}\right)
$$

satisfies

$$
\begin{aligned}
D(u+v)(0) & =0, \\
D^{2}(u+v)(0) & <0 .
\end{aligned}
$$

These two conditions imply the origin is a strict local maximum point for $u+v$, and so $u+v$ fails to satisfy the maximum principle on $V$ whenever $V \in \mathscr{B}(U)$ contains the origin and is sufficiently small (using that $v$ has the desired form (5) as soon as $V$ is small enough to avoid the singularities at $\xi_{1}, \ldots, \xi_{n}$ ). This failure of the maximum principle establishes the contrapositive of part (a) of the theorem.

The next portion of the proof, in which we choose $a_{m}$ and $\xi_{m}$, will appear unmotivated and should really be read backwards from the end. But in the interests of clear logic, we build the proof carefully from the ground up.

Write

$$
D^{2} u=\left[D_{j} D_{k} u\right]_{j, k=1}^{n}
$$

for the Hessian matrix of $u$, so that $D^{2} u$ is real and symmetric at each $x$. In particular the Hessian $D^{2} u(0)$ at the origin has $n$ real eigenvalues (counted 
with respect to multiplicity), which we denote by $\lambda_{1}, \ldots, \lambda_{n}$. Choose a number $\lambda>0$ large enough that $\lambda>\lambda_{m}$ for all $m$, or $1-\lambda_{m} / \lambda>0$ for all $m$. Notice that

$$
\begin{aligned}
\sum_{m=1}^{n} \frac{1-\lambda_{m} / \lambda}{n}=1-\left(\lambda_{1}+\cdots+\lambda_{n}\right) / \lambda n & =1-\operatorname{Trace} D^{2} u(0) / \lambda n \\
& =1-\Delta u(0) / \lambda n>1
\end{aligned}
$$

since $\Delta u(0)<0$. Therefore it is possible to choose numbers $\alpha_{1}, \ldots, \alpha_{n}>0$ such that $\sum_{m=1}^{n} \alpha_{m}=1$ and

$$
\frac{1-\lambda_{m} / \lambda}{n}>\alpha_{m}, \quad m=1, \ldots, n .
$$

These $n$ inequalities imply the diagonal matrix inequality

$$
E-\lambda I+\sum_{m=1}^{n} \alpha_{m} \lambda\left(n e_{m} e_{m}^{T}\right)<0,
$$

where $E$ is the diagonal matrix of eigenvalues, with entries $\lambda_{1}, \ldots, \lambda_{n}$, and where $e_{m}=(0, \ldots, 1, \ldots, 0)^{T}$ is the $m^{\text {th }}$ unit column vector. Hence

$$
E+\sum_{m=1}^{n} \alpha_{m} \lambda\left(-I+n q_{m} q_{m}^{T}\right)<0
$$

whenever $q_{1}, \ldots, q_{n}$ are vectors sufficiently close to $e_{1}, \ldots, e_{n}$, respectively, where we have used also here that $\sum_{m=1}^{n} \alpha_{m}=1$.

Now take $p_{1}, \ldots, p_{n}$ to be an orthonormal collection of eigenvectors of $D^{2} u(0)$ corresponding to the eigenvalues $\lambda_{1}, \ldots, \lambda_{n}$, so that $P^{T} D^{2} u(0) P=E$ where $P=\left[p_{1} \cdots p_{n}\right]$ is the matrix with the $p_{m}$ as its columns. We may suppose $-p_{m}^{T} D u(0) \geq 0$ for each $m$, just by replacing $p_{m}$ with $-p_{m}$ if necessary. That is,

$$
-P^{T} \mathrm{Du}(0) \cdot e_{m} \geq 0, \quad m=1, \ldots, n .
$$

Geometrically, this says the vector $-P^{T} D u(0)$ lies in the closed first quadrant of $\mathrm{R}^{n}$, and is nonzero (since $D u(0) \neq 0$ ). Clearly an orthonormal system $q_{1}, \ldots, q_{n}$ exists such that

$$
-P^{T} D u(0) \cdot q_{m}>0, \quad m=1, \ldots, n,
$$

and that the $q_{1}, \ldots, q_{n}$ are a small enough perturbation of $e_{1}, \ldots, e_{n}$ for (8) to hold. 
Write $z_{1}, \ldots, z_{n}$ for the columns of $P Q$, meaning $P Q=\left[z_{1} \cdots z_{n}\right]$, and let

$$
\xi_{m}=-\frac{\left(P^{T} D u(0) \cdot q_{m}\right)}{\alpha_{m} \lambda} z_{m},
$$

so that the $\xi_{m}$ are all distinct (since the $z_{m}$ are orthonormal) and

$$
\xi_{m}=\left|\xi_{m}\right| z_{m} \quad \text { where } \quad\left|\xi_{m}\right|=-\frac{\left(P^{T} D u(0) \cdot q_{m}\right)}{\alpha_{m} \lambda}>0 .
$$

We will show the singularities $\xi_{m}$ lie in $U$ whenever $\lambda$ is chosen sufficiently large. Clearly $\frac{1-\lambda_{m} / \lambda}{n}<\frac{2}{2 n-1}$ for all large $\lambda$, and for such $\lambda$ our choice of $\alpha_{m}<\frac{1-\lambda_{m} / \lambda}{n}$ guarantees $\alpha_{m}<\frac{2}{2 n-1}$ for all $m$. Thus

$$
\alpha_{\ell}=1-\sum_{m \neq \ell} \alpha_{m}>1-(n-1) \frac{2}{2 n-1}=\frac{1}{2 n-1}
$$

for each $\ell$. Hence

$$
\left|\xi_{\ell}\right|=-\frac{P^{T} D u(0) \cdot q_{\ell}}{\alpha_{\ell} \lambda}<\frac{\left|P^{T} D u(0)\right|}{\frac{1}{2 n-1} \lambda} \rightarrow 0
$$

as $\lambda \rightarrow \infty$, so that by choosing $\lambda$ sufficiently large we ensure the singularities $\xi_{m}$ all lie close to $x_{0}=0$ and inside $U$.

Now define

$$
\begin{aligned}
a_{m} & =-\left(D u(0) \cdot z_{m}\right)\left|\xi_{m}\right|^{n-1} & & \\
& =-\left(P^{T} D u(0) \cdot q_{m}\right)\left|\xi_{m}\right|^{n-1} & & \text { since } z_{m}=P q_{m} \\
& =\alpha_{m} \lambda\left|\xi_{m}\right|^{n} & & \text { by (10) } \\
& >0 . & &
\end{aligned}
$$

This completes our definitions. Now to prove (6) and (7), we compute

$$
\begin{aligned}
v_{x_{j}}(x) & =\sum_{m=1}^{n} a_{m}(-1) \frac{x_{j}-\left(\xi_{m}\right)_{j}}{\left|x-\xi_{m}\right|^{n}}, \\
v_{x_{j}}(0) & =\sum_{m=1}^{n} a_{m} \frac{\left(\xi_{m}\right)_{j}}{\left|\xi_{m}\right|^{n}} \\
v_{x_{j} x_{k}}(0) & =\sum_{m=1}^{n} a_{m} \frac{1}{\left|\xi_{m}\right|^{n}}\left(-\delta_{j k}+n \frac{\left(\xi_{m}\right)_{j}\left(\xi_{m}\right)_{k}}{\left|\xi_{m}\right|^{2}}\right),
\end{aligned}
$$


and hence

$$
\begin{aligned}
D v(0) & =\sum_{m=1}^{n} \alpha_{m} \lambda \xi_{m}=\sum_{m=1}^{n} \frac{a_{m}}{\left|\xi_{m}\right|^{n-1}} z_{m}, \\
D^{2} v(0) & =\sum_{m=1}^{n} \frac{a_{m}}{\left|\xi_{m}\right|^{n}}\left(-I+n z_{m} z_{m}^{T}\right),
\end{aligned}
$$

since $z_{m}=\xi_{m} /\left|\xi_{m}\right|$. Using (12) and the definition of $a_{m}$, we find

$$
D v(0)=-\sum_{m=1}^{n}\left(D u(0) \cdot z_{m}\right) z_{m}=-D u(0)
$$

because the $z_{m}$ form an orthonormal basis (notice $P$ and $Q$ are orthogonal matrices). Thus $D(u+v)(0)=0$, which is (6).

Conjugating the Hessian inequality (7) with $P$ shows that it is equivalent to

$$
P^{T}\left\{D^{2} u(0)+D^{2} v(0)\right\} P<0 .
$$

Then substituting in the formula (13) for the Hessian of $v$, and invoking relation (11), reduces this last inequality to (8) (recalling $P^{T} z_{m}=q_{m}$ ). This proves (7).

Part (b). It remains to prove $u$ is subharmonic if $u-v$ (instead of $u+v$ ) satisfies the maximum principle on $V$ whenever $V \in \mathscr{B}(U)$ and $v$ has the form (5). The goal is now to prove (6) and (7) with $v$ replaced by $-v$. Modifying the proof above, we choose $\lambda>0$ large enough that $\lambda>-\lambda_{m}$ for all $m$, or $1+\lambda_{m} / \lambda>0$ for all $m$. Then

$$
\sum_{m=1}^{n} \frac{1+\lambda_{m} / \lambda}{n}=1+\Delta u(0) / \lambda n<1
$$

since $\Delta u(0)<0$. Therefore it is possible to choose numbers $\alpha_{1}, \ldots, \alpha_{n}>0$ such that $\sum_{m=1}^{n} \alpha_{m}=1$ and

$$
\frac{1+\lambda_{m} / \lambda}{n}<\alpha_{m}, \quad m=1, \ldots, n .
$$

Hence the analogue of (8) is

$$
E-\sum_{m=1}^{n} \alpha_{m} \lambda\left(-I+n q_{m} q_{m}^{T}\right)<0 .
$$

Continuing with the proof like earlier, we may suppose $P^{T} D u(0) \cdot e_{m} \geq 0$ for each $m$. Thus an orthonormal system $q_{1}, \ldots, q_{n}$ exists such that $P^{T} D u(0)$. 
$q_{m}>0$ for each $m$, and that the $q_{1}, \ldots, q_{m}$ are a small enough perturbation of $e_{1}, \ldots, e_{m}$ for (14) to hold.

Letting

$$
\xi_{m}=\frac{\left(P^{T} D u(0) \cdot q_{m}\right)}{\alpha_{m} \lambda} z_{m},
$$

one finds

$$
\xi_{m}=\left|\xi_{m}\right| z_{m} \quad \text { where } \quad\left|\xi_{m}\right|=\frac{\left(P^{T} D u(0) \cdot q_{m}\right)}{\alpha_{m} \lambda}>0 .
$$

To show the singularities $\xi_{m}$ lie in $U$ whenever $\lambda$ is chosen sufficiently large, just observe

$$
\alpha_{m} \lambda>\frac{\lambda+\lambda_{m}}{n} \rightarrow \infty
$$

as $\lambda \rightarrow \infty$, which implies by (15) that $\left|\xi_{m}\right| \rightarrow 0$ as $\lambda \rightarrow \infty$. (Notice this part of the proof is easier than in part (a)).

To complete the proof, only a few more sign changes need be made: define $a_{m}=\left(D u(0) \cdot z_{m}\right)\left|\xi_{m}\right|^{n-1}=\alpha_{m} \lambda\left|\xi_{m}\right|^{n}>0$, and use (12) and the definition of $a_{m}$ to show $D v(0)=D u(0)$, giving $D(u-v)(0)=0$. Finally, after conjugating the Hessian inequality with $P$ and changing $v$ to $-v$, the desired inequality is $P^{T}\left\{D^{2} u(0)-D^{2} v(0)\right\} P<0$, which we prove like before.

\subsection{Proof of Corollary 3.2}

Let $u: U \rightarrow[-\infty, \infty)$ be upper semicontinuous with $u \not \equiv-\infty$. We will prove only part (a), since the proof of part (b) is the same except with $v$ changed to $-v$.

To prove the contrapositive of part (a), suppose $u$ is not subharmonic in $U$. Then $u$ is not a viscosity subsolution of Laplace's equation in $U$, by the equivalence of viscosity subsolutions and the usual potential theoretic subharmonic functions (see [7, Theorem 2.7] with $p=2$, and the references therein).

Hence by definition of viscosity subsolution (see [7, Definition 2.5]), there exists a point $x_{0} \in U$ and a function $\phi \in C^{2}(U)$ such that $u\left(x_{0}\right)=\phi\left(x_{0}\right)$, $u(x)<\phi(x)$ for all $x \in U \backslash\left\{x_{0}\right\}$, and $D \phi\left(x_{0}\right) \neq 0$ and $\Delta \phi\left(x_{0}\right)<0$. By applying the proof of Theorem 3.1 to the function $\phi$, we find a function $v$ of the form (5) such that

$$
D(\phi+v)\left(x_{0}\right)=0, \quad D^{2}(\phi+v)\left(x_{0}\right)<0 .
$$

Then clearly $u+v=(u-\phi)+(\phi+v)$ has a strict local maximum at $x_{0}$, so that $u+v$ fails to satisfy the maximum principle on all sufficiently small domains $V \in \mathscr{B}(U)$ contiaining the point $x_{0} \in V$. That is, condition (a) in Theorem 3.1 fails, completing the proof of the contrapositive. 


\section{Subtemperatures}

\subsection{The characterization}

Now we characterize subtemperatures. As explained later, our results improve on recent work of Crandall and Wang [4].

Write the fundamental solution of the heat (or diffusion) operator in $\mathrm{R}^{n+1}$, $n \geq 1$, as

$$
K(x, t)=\frac{1}{(4 \pi t)^{n / 2}} e^{-|x|^{2} / 4 t}, \quad x \in \mathrm{R}^{n}, t>0 .
$$

Call a set $W \subset \mathbf{R}^{n+1}$ a cylinder if it has the form $W=V \times\left(t_{1}, t_{2}\right)$ where $V \subset \mathbf{R}^{n}$ is open and $t_{1}<t_{2}$.

Let $U$ be an open set in $\mathrm{R}^{n+1}, n \geq 1$. Suppose $\mathscr{B}_{p}(U)$ (where the " $p$ " stands for parabolic) is a collection of cylinders contained in $U$ such that for each $(x, t) \in U$ and $\varepsilon>0$ there exists a cylinder $W=V \times\left(t_{1}, t\right)$ in $\mathscr{B}_{p}(U)$ with $x \in V \subset B(x, \varepsilon)$ and $0<t-t_{1}<\varepsilon$.

For example, $\mathscr{B}_{p}(U)$ could consist of all cylinders in which $V$ is a ball with radius in $\left\{1, \frac{1}{2}, \frac{1}{3}, \ldots\right\}$ and with $\bar{W} \subset U$.

Write $C^{2,1}(U)$ for the class of functions with two spatial derivatives and one temporal derivative in $U$, with all these derivatives being continuous. For $u \in C^{2,1}(U)$ to be called a subtemperature means that $\Delta u \geq u_{t}$, where $\Delta$ denotes the Laplacian in the spatial variables. In Corollary 4.2 below one should instead use the potential theoretic definition of a subtemperature (see [7, Definition 4.4] with $p=2$ ).

With these definitions, we can now state our characterization of subtemperatures.

Theorem 4.1. Let $u: U \rightarrow \mathbf{R}$ where $U$ is an open set in $\mathbf{R}^{n+1}, n \geq 1$, with associated collection of cylinders $\mathscr{B}_{p}(U)$ as above. Suppose $u \in C^{2,1}(U)$. Assume either

(a) $u+w$ satisfies the parabolic maximum principle on $W$ whenever $W \in$ $\mathscr{B}_{p}(U)$ and $w$ has the form

$$
w(x, t)=\sum_{m=1}^{n} b_{m} K\left(x-\xi_{m}, t-\tau_{m}\right)
$$

for some positive real numbers $b_{1}, \ldots, b_{n}$, and some distinct points $\left(\xi_{m}, \tau_{m}\right) \in U$ with $\tau_{m}<t_{1}$; or else

(b) $u-w$ satisfies the parabolic maximum principle on $W$ whenever $W \in$ $\mathscr{B}_{p}(U)$ and $w$ has the form (16), and also $u_{t} \leq 0$ in every subregion of $U$ on which $u$ is independent of $x$.

Then $u$ is a subtemperature in $U$. 
CoROLlaRy 4.2. Theorem 4.1(a) still holds if the hypotheses $u: U \rightarrow \mathbf{R}$ and $u \in C^{2,1}(U)$ are weakened to $u: U \rightarrow[-\infty, \infty)$ being upper semicontinuous with $u$ finite in a dense subset of $U$.

The parabolic maximum principle for a function $\psi$ means $\max _{\bar{W}} \psi=$ $\max _{\partial_{p} W} \psi$, where

$$
\partial_{p} W=\left(V \times\left\{t_{1}\right\}\right) \cup\left(\partial V \times\left[t_{1}, t_{2}\right]\right)
$$

denotes the parabolic boundary of $W$.

REMARKs. 1. The function $w$ in formula (16) is a positive linear combination of $n$ fundamental solutions with singularities occurring before the time $t_{1}$ at which $\bar{W}$ begins. Clearly $w$ is a temperature on $\bar{W}$ (meaning $\Delta w=w_{t}$ there). Thus the converse of Theorem 4.1 is easy: if a function $u$ is a subtemperature on $U$ then $u \pm w$ is a subtemperature on $\bar{W}$, and so $u \pm w$ satisfies the parabolic maximum principle there.

2. One cannot remove the requirement in condition (b) that $u_{t} \leq 0$ in every subregion of $U$ on which $u$ is independent of $x$. For example in dimension $n=$ 1 suppose $u(x, t)=t$, which is not a subtemperature. Notice $u$ is independent of $x$ with $u_{t}>0$, so that $u$ fails the second requirement of (b). However $u$ does satisfy the first requirement of (b); indeed $u-w=t-b_{1} K(x-$ $\left.\xi_{1}, t-\tau_{1}\right)$ satisfies the parabolic maximum principle on every cylinder $W=$ $V \times\left(t_{1}, t_{2}\right), \tau_{1}<t_{1}$, because the Gaussian $x \mapsto e^{-x^{2}}$ has no local minimum.

3. Corollary 4.2 covers only part (a) of Theorem 4.1. We believe part (b) should hold also, when $u: U \rightarrow[-\infty, \infty)$ is upper semicontinuous and finite in a dense subset of $U$, but we do not see how to prove this.

4. Crandall and Wang [4, Theorem 3] recently proved Theorem 4.1(a) and Corollary 4.2, except that they needed $n+1$ terms in the definition of $w$, whereas we need only $n$ terms. The first reason we do better here is that when proving Theorem 4.1(a), we perturb $\left(x_{0}, t_{0}\right)$ so as to get $D_{x} u\left(x_{0}, t_{0}\right) \neq 0$ : this is possible except when $u$ depends only on $t$, which we treat separately. Once one has $D_{x} u\left(x_{0}, t_{0}\right) \neq 0$, one needs only $n$ terms in $w$ to complete the proof (as Crandall and Wang also observed). The second reason we need only $n$ terms is that when proving Corollary 4.2 we use a nominally weaker notion of viscosity subsolution (a notion going back to Ohnuma and Sato, thence to Ishii and Souganidis), which gives more control over the test function $\phi$ at points where its spatial gradient vanishes. This additional control at points where $D_{x} \phi\left(x_{0}, t_{0}\right)=0$ enables us to again use only $n$ terms, when constructing $w$.

5. We learned of Crandall and Wang's work only after writing this paper. Our methods are very similar to theirs, due to the common starting point (the subharmonic characterization Theorem 1.1 by Crandall and Zhang). 
6. Our proof of Theorem 4.1(a) is simpler than the proof of Crandall and Wang in that it avoids the "non-generic" case of [4, p. 8] (the case with $\tilde{S}$ ), by means of our perturbation of the standard basis vector $e_{m}$ to the vector $q_{m}$, in the proof below.

7. Crandall and Wang do not localize their singularities, although presumably they could modify their proof a little to enforce $\left(\xi_{m}, \tau_{m}\right) \in U$, like in our work.

8. Theorem 4.1(b), in which we treat $u-w$ instead of $u+w$, has no analogue in Crandall and Wang's paper.

9. Fundamental solutions are not the only choice for characterizing subtemperatures by means of the maximum principle. Indeed the simpler class of functions $w(x, t)=\frac{1}{2} x^{T} A x+p \cdot x+t$ Trace $A$ was shown to suffice by Crandall and Wang [4, Remark 6], where $A$ is a symmetric $n \times n$ matrix and $p \in \mathrm{R}^{n}$.

Proof of Theorem 4.1. Part (a). We establish the contrapositive. So assume $u$ is not a subtemperature, meaning $\Delta u<u_{t}$ at some point $\left(x_{0}, t_{0}\right) \in U$. Assume $D_{x} u\left(x_{0}, t_{0}\right) \neq 0$, and consider later the case $D_{x} u\left(x_{0}, t_{0}\right)=0$. (Here $D_{x}$ denotes the spatial gradient operator, taken with respect to the $x$-variables and keeping $t$ fixed.)

By a translation we may suppose $\left(x_{0}, t_{0}\right)=(0,0)$, so that $\Delta u(0,0)<$ $u_{t}(0,0)$ and $D_{x} u(0,0) \neq 0$. Define $\varepsilon>0$ by

$$
\varepsilon=u_{t}(0,0)-\Delta u(0,0) .
$$

We will choose $b_{m}>0, \xi_{m} \in \mathrm{R}^{n}$ distinct and $\tau_{m}<0$ in such a way that

$$
w(x, t)=\sum_{m=1}^{n} b_{m} K\left(x-\xi_{m}, t-\tau_{m}\right)
$$

satisfies

$$
\begin{gathered}
D_{x}(u+w)(0,0)=0, \\
D_{x}^{2}(u+w)(0,0)<0, \\
(u+w)_{t}(0,0)>0 .
\end{gathered}
$$

These imply the origin is a strict local maximum point for $u+w$ with respect to any sufficiently small cylinder $W=V \times\left(t_{1}, 0\right)$ having $0 \in V$ and $t_{1}<0$. Thus $u+w$ does not satisfy the parabolic maximum principle, whenever this cylinder $W$ belongs to $\mathscr{B}_{p}(U)$ and is sufficiently small (noting that $w$ has the desired form (16) provided $t_{1}>\max _{m} \tau_{m}$ ). This failure of the maximum principle establishes the contrapositive of part (a) of the theorem. 
Like in the proof of Theorem 3.1, we take $\lambda_{1}, \ldots, \lambda_{n}$ to be the eigenvalues of the spatial Hessian $D_{x}^{2} u(0,0)$ at the origin, and we choose $\lambda>0$ large enough that $\lambda>\lambda_{m}$ for all $m$. Then by summing,

$$
n \lambda-\left(\lambda_{1}+\cdots+\lambda_{n}\right)>0 .
$$

Hence we may choose a number $v>0$ satisfying

$$
n-\left(\lambda_{1}+\cdots+\lambda_{n}\right) / \lambda>v>n-\left(\lambda_{1}+\cdots+\lambda_{n}+\varepsilon\right) / \lambda .
$$

Thus $v \rightarrow n$ as $\lambda \rightarrow \infty$.

Notice that

$$
\sum_{m=1}^{n} \frac{1-\lambda_{m} / \lambda}{v}=\frac{1}{v}\left(n-\frac{\lambda_{1}+\cdots+\lambda_{n}}{\lambda}\right)>1
$$

by choice of $\nu$. Therefore it is possible to choose numbers $\beta_{1}, \ldots, \beta_{n}>0$ such that $\sum_{m=1}^{n} \beta_{m}=1$ and

$$
\frac{1-\lambda_{m} / \lambda}{v}>\beta_{m}, \quad m=1, \ldots, n .
$$

These $n$ inequalities imply the diagonal matrix inequality

$$
E-\lambda I+\sum_{m=1}^{n} \beta_{m} \lambda\left(v e_{m} e_{m}^{T}\right)<0,
$$

where $E$ is the diagonal matrix of eigenvalues and the $e_{m}$ are the standard basis vectors. Hence

$$
E+\sum_{m=1}^{n} \beta_{m} \lambda\left(-I+v q_{m} q_{m}^{T}\right)<0
$$

whenever $q_{1}, \ldots, q_{n}$ are vectors sufficiently close to $e_{1}, \ldots, e_{n}$, respectively, where we have used also here that $\sum_{m=1}^{n} \beta_{m}=1$. Write $Q$ for the matrix with the $q_{m}$ as its column vectors.

As before, we take $P=\left[p_{1} \cdots p_{n}\right]$ to be an orthogonal matrix whose columns $p_{m}$ are eigenvectors of $D_{x}^{2} u(0,0)$ corresponding to the eigenvalues $\lambda_{1}, \ldots, \lambda_{n}$, so that $P^{T} D_{x}^{2} u(0,0) P=E$. We may suppose $-p_{m}^{T} D_{x} u(0,0) \geq 0$ for each $m$, or

$$
-P^{T} D_{x} u(0,0) \cdot e_{m} \geq 0, \quad m=1, \ldots, n .
$$


This means the vector $-P^{T} D_{x} u(0,0)$ lies in the closed first quadrant of $\mathbf{R}^{n}$, and is nonzero (since $D_{x} u(0,0) \neq 0$ ). Again we may choose an orthonormal system $q_{1}, \ldots, q_{n}$ such that

$$
-P^{T} D_{x} u(0,0) \cdot q_{m}>0, \quad m=1, \ldots, n,
$$

and that the $q_{1}, \ldots, q_{n}$ are a small enough perturbation of $e_{1}, \ldots, e_{n}$ for (22) to hold.

Once more write $P Q=\left[z_{1} \cdots z_{n}\right]$, and let

$$
\xi_{m}=-\frac{\left(P^{T} D_{x} u(0,0) \cdot q_{m}\right)}{\beta_{m} \lambda} z_{m},
$$

so that the $\xi_{m}$ are all distinct and

$$
\xi_{m}=\left|\xi_{m}\right| z_{m} \quad \text { where } \quad\left|\xi_{m}\right|=-\frac{\left(P^{T} D_{x} u(0,0) \cdot q_{m}\right)}{\beta_{m} \lambda}>0 .
$$

Put

$$
\tau_{m}=-\frac{\left|\xi_{m}\right|^{2}}{2 v}<0
$$

and define

$$
b_{m}=-\left(D_{x} u(0,0) \cdot z_{m}\right)\left(-2 \tau_{m}\right) /\left(\left|\xi_{m}\right| K\left(-\xi_{m},-\tau_{m}\right)\right) .
$$

Notice

$$
\begin{aligned}
\frac{b_{m}}{2\left(-\tau_{m}\right)}\left|\xi_{m}\right| K\left(-\xi_{m},-\tau_{m}\right) & =-\left(D_{x} u(0,0) \cdot z_{m}\right) \\
& =-\left(P^{T} D_{x} u(0,0) \cdot q_{m}\right) \quad \text { since } z_{m}=P q_{m} \\
& =\beta_{m} \lambda\left|\xi_{m}\right| \\
& >0
\end{aligned}
$$

This shows $b_{m}>0$.

We must show $\left(\xi_{m}, \tau_{m}\right) \in U$ whenever $\lambda$ is chosen sufficiently large. For this it is enough to show that $\left|\xi_{m}\right|$ and $\left|\tau_{m}\right|$ approach zero as $\lambda \rightarrow \infty$, since $\left(x_{0}, t_{0}\right)=(0,0)$ lies in $U$.

Since $v \rightarrow n$ as $\lambda \rightarrow \infty$, we see $\frac{1-\lambda_{m} / \lambda}{v}<\frac{2}{2 n-1}$ for all large $\lambda$. Then our choice of $\beta_{m}<\frac{1-\lambda_{m} / \lambda}{v}$ guarantees that $\left|\xi_{\ell}\right| \rightarrow 0$ as $\lambda \rightarrow \infty$ by arguing like in the proof of Theorem 3.1. Hence $\tau_{m}=-\left|\xi_{m}\right|^{2} / 2 v \rightarrow 0$ as $\lambda \rightarrow \infty$ as we wanted. 
Now to prove (18)-(20), we first compute

$$
\begin{aligned}
w_{x_{j}}(x, t) & =\sum_{m=1}^{n} b_{m}(-1) \frac{x_{j}-\left(\xi_{m}\right)_{j}}{2\left(t-\tau_{m}\right)} K\left(x-\xi_{m}, t-\tau_{m}\right), \\
w_{x_{j}}(0,0) & =\sum_{m=1}^{n} b_{m} \frac{\left(\xi_{m}\right)_{j}}{2\left(-\tau_{m}\right)} K\left(-\xi_{m},-\tau_{m}\right), \\
w_{x_{j} x_{k}}(0,0) & =\sum_{m=1}^{n} b_{m} \frac{1}{2\left(-\tau_{m}\right)}\left(-\delta_{j k}+\frac{\left(\xi_{m}\right)_{j}\left(\xi_{m}\right)_{k}}{2\left(-\tau_{m}\right)}\right) K\left(-\xi_{m},-\tau_{m}\right) .
\end{aligned}
$$

Then (26) implies

$$
D_{x} w(0,0)=-\sum_{m=1}^{n}\left(D_{x} u(0,0) \cdot z_{m}\right) z_{m}
$$

by using (24) and $\xi_{m}=\left|\xi_{m}\right| z_{m}$, while (27) implies

$$
D_{x}^{2} w(0,0)=\sum_{m=1}^{n} \beta_{m} \lambda\left(-I+v z_{m} z_{m}^{T}\right)
$$

by using (25) and $\xi_{m}=\left|\xi_{m}\right| z_{m}$ and $v=\left|\xi_{m}\right|^{2} / 2\left(-\tau_{m}\right)$.

Now (28) shows $D_{x} w(0,0)=-D_{x} u(0,0)$ because the $z_{m}$ form an orthonormal basis. Thus $D_{x}(u+w)(0,0)=0$, which is (18).

Conjugating the Hessian inequality (19) with $P$ shows it is equivalent to

$$
P^{T}\left\{D_{x}^{2} u(0,0)+D_{x}^{2} w(0,0)\right\} P<0 .
$$

Then substituting in the formula (29) for the Hessian of $w$ reduces this inequality to (22) (since $P^{T} z_{m}=q_{m}$ ), thus completing the proof of (19).

Lastly, to prove (20) we observe

$$
\begin{aligned}
& (u+w)_{t}(0,0) \\
& \quad=\varepsilon+\Delta(u+w)(0,0) \quad \text { by choice of } \varepsilon \\
& \quad=\varepsilon+\operatorname{Trace} D_{x}^{2}(u+w)(0,0) \\
& \quad=\varepsilon+\operatorname{Trace}\left[P^{T} D_{x}^{2} u(0,0) P\right]+\operatorname{Trace}\left[(P Q)^{T} D_{x}^{2} w(0,0)(P Q)\right] \\
& \quad=\varepsilon+\operatorname{Trace} E+\sum_{m=1}^{n} \beta_{m} \lambda \operatorname{Trace}\left[-I+v e_{m} e_{m}^{T}\right] \\
& \quad=\varepsilon+\left(\lambda_{1}+\cdots+\lambda_{n}\right)+\lambda(-n+v) \\
& \quad>0
\end{aligned}
$$


by definition of $v$ in (21). This completes the proof in the case where $D_{x} u\left(x_{0}, t_{0}\right)$ $\neq 0$.

Now return to the beginning of the proof and consider the case where $\Delta u<u_{t}$ and $D_{x} u=0$ at some point $\left(x_{0}, t_{0}\right) \in U$. In fact, we can suppose $D_{x} u \equiv 0$ on a neighborhood of $\left(x_{0}, t_{0}\right)$, because otherwise we could instead take $\left(x_{0}, t_{0}\right)$ to be some nearby point at which $D_{x} u \neq 0$ and $\Delta u<u_{t}$, and then apply the previous case. But if $D_{x} u \equiv 0$ near $\left(x_{0}, t_{0}\right)$, then the Hessian vanishes too: $D_{x}^{2} u \equiv 0$ near $\left(x_{0}, t_{0}\right)$.

By a translation we can suppose $\left(x_{0}, t_{0}\right)=(0,0)$, so that $u_{t}(0,0)>$ $\Delta u(0,0)=0$. Choose $\xi_{m}=0$ and $\tau_{m}<0$ for all $m$, in the definition (17) of $w$, with the $\tau_{m}$ chosen to be distinct and so small that $\left(\xi_{m}, \tau_{m}\right) \in U$. And then choose $b_{m}>0$ to be so small that $(u+w)_{t}(0,0)>0$, meaning (20) holds. Notice $D_{x} w(0,0)=0$ by (26) (since the Gaussians in $w$ are all centered at the spatial origin $\left.\xi_{m}=0\right)$, so that $D_{x}(u+w)(0,0)=0$, which gives (18).

Further,

$$
\begin{aligned}
D_{x}^{2}(u+w)(0,0)=D_{x}^{2} w(0,0) & =-\sum_{m=1}^{n} \frac{b_{m}}{2\left(-\tau_{m}\right)} K\left(0,-\tau_{m}\right) I \\
& <0
\end{aligned}
$$

which gives (19). Now (18)-(20) suffice to show that the parabolic maximum principle fails for some $W$, just like before.

Part (b). Again we establish the contrapositive. Assume $u$ is not a subtemperature, meaning $\Delta u<u_{t}$ at some point $\left(x_{0}, t_{0}\right) \in U$.

If $D_{x} u \equiv 0$ on a neighborhood of $\left(x_{0}, t_{0}\right)$ then $0 \equiv \Delta u<u_{t}$ near $\left(x_{0}, t_{0}\right)$, and so condition (b) fails as desired. Thus we can assume $D_{x} u \neq \equiv 0$ on every neighborhood of $\left(x_{0}, t_{0}\right)$. Hence by perturbing the point $\left(x_{0}, t_{0}\right)$ slightly, we can suppose $D_{x} u\left(x_{0}, t_{0}\right) \neq 0$.

Now proceed like in the proof of part (a), except with the following changes. Choose $\lambda>0$ large enough that $\lambda>-\lambda_{m}$ for all $m$. Choose $v>0$ to satisfy

$$
n+\left(\lambda_{1}+\cdots+\lambda_{n}\right) / \lambda<v<n+\left(\lambda_{1}+\cdots+\lambda_{n}+\varepsilon\right) / \lambda
$$

so that

$$
\sum_{m=1}^{n} \frac{1+\lambda_{m} / \lambda}{v}=\frac{1}{v}\left(n+\frac{\lambda_{1}+\cdots+\lambda_{n}}{\lambda}\right)<1
$$

Then it is possible to choose the $\beta_{m}>0$ to satisfy $\sum_{m=1}^{n} \beta_{m}=1$ and

$$
\frac{1+\lambda_{m} / \lambda}{v}<\beta_{m}, \quad m=1, \ldots, n,
$$


which leads to the matrix inequality

$$
E-\sum_{m=1}^{n} \beta_{m} \lambda\left(-I+v q_{m} q_{m}^{T}\right)<0
$$

whenever the $q_{m}$ are sufficiently close to the $e_{m}$.

We may suppose $P^{T} D_{x} u(0,0) \cdot e_{m} \geq 0$ for each $m$, and then choose the $q_{m}$ to be an orthonormal system with $P^{T} D_{x} u(0,0) \cdot q_{m}>0$ for each $m$ (using here that $D_{x} u(0,0) \neq 0$ ) and with the $q_{m}$ sufficiently close to the $e_{m}$ for the previous paragraph to apply.

Letting

$$
\xi_{m}=\frac{\left(P^{T} D_{x} u(0,0) \cdot q_{m}\right)}{\beta_{m} \lambda} z_{m},
$$

one finds the $\xi_{m}$ are all distinct and $\xi_{m}=\left|\xi_{m}\right| z_{m}$ where $\left|\xi_{m}\right|=\left(P^{T} D_{x} u(0,0)\right.$. $\left.q_{m}\right) /\left(\beta_{m} \lambda\right)>0$.

Put $\tau_{m}=-\frac{\left|\xi_{m}\right|^{2}}{2 v}<0$ and $b_{m}=\left(D_{x} u(0,0) \cdot z_{m}\right)\left(-2 \tau_{m}\right) /\left(\left|\xi_{m}\right| K\left(-\xi_{m},-\tau_{m}\right)\right)$, so that $b_{m}>0$ like before.

To show $\left|\xi_{m}\right| \rightarrow 0$ as $\lambda \rightarrow \infty$ it suffices to observe

$$
\beta_{m} \lambda>\frac{\lambda+\lambda_{m}}{v} \rightarrow \infty \quad \text { as } \quad \lambda \rightarrow \infty
$$

(This part of the proof is easier than in part (a).)

With these preliminaries, one can verify that $w$ satisfies

$$
\begin{aligned}
& D_{x}(u-w)(0,0)=0, \\
& D_{x}^{2}(u-w)(0,0)<0 .
\end{aligned}
$$

Furthermore

$$
\begin{aligned}
(u-w)_{t}(0,0) & =\varepsilon+\Delta(u-w)(0,0) \quad \text { by choice of } \varepsilon \\
& =\varepsilon+\left(\lambda_{1}+\cdots+\lambda_{n}\right)-\lambda(-n+v) \\
& >0
\end{aligned}
$$

by definition of $v$. From these last three inequalities, we deduce condition (b) fails for some small cylinder $W$. 


\subsection{Proof of Corollary 4.2}

Let $u: U \rightarrow[-\infty, \infty)$ be upper semicontinuous with $u$ finite in a dense subset of $U$.

To prove the contrapositive of part (a), suppose $u$ is not a subtemperature in $U$. Then $u$ is not a relaxed viscosity subsolution of the heat equation in $U$, by the equivalence between the relaxed viscosity subsolutions and the potential theoretic subtemperatures (see [7, Theorem 4.6] and [7, p. 716], with $p=2$; and note the references to earlier work, especially that of Ohnuma and Sato [10]).

Hence by definition of relaxed viscosity subsolution (see [7, Definition 4.17]), there exists a function $\phi \in C^{2}(U)$ and a local maximum point $\left(x_{0}, t_{0}\right) \in$ $U$ of $u-\phi$ such that $\Delta \phi\left(x_{0}, t_{0}\right)<\phi_{t}\left(x_{0}, t_{0}\right)$ and such that if $D_{x} \phi\left(x_{0}, t_{0}\right)=0$ then $D_{x}^{2} \phi\left(x_{0}, t_{0}\right)=0$. (We have used here the definition of "admissibility" for $\phi$, from [7, Definition 4.16], which ensures that if $D_{x} \phi\left(x_{0}, t_{0}\right)=0$ then $\phi\left(x, t_{0}\right)-\phi\left(x_{0}, t_{0}\right)=o\left(\left|x-x_{0}\right|^{2}\right)$ and hence that the Hessian of $\phi$ vanishes at $\left(x_{0}, t_{0}\right)$.)

If $D_{x} \phi\left(x_{0}, t_{0}\right) \neq 0$ then we can apply the first part of the proof of Theorem 4.1(a) to the function $\phi$, while if $D_{x} \phi\left(x_{0}, t_{0}\right)=0$ then $D_{x}^{2} \phi\left(x_{0}, t_{0}\right)=0$ by assumption and so we can apply the second part of the proof of Theorem 4.1(a) to $\phi$. Either way we obtain a function $w$ of the form (16) such that

$$
D_{x}(\phi+w)\left(x_{0}, t_{0}\right)=0, \quad D_{x}^{2}(\phi+w)\left(x_{0}, t_{0}\right)<0, \quad(\phi+w)_{t}\left(x_{0}, t_{0}\right)>0 .
$$

Then $u+w=(u-\phi)+(\phi+w)$ has a strict local maximum at $\left(x_{0}, t_{0}\right)$, relative to times $t<t_{0}$. Hence $u+w$ fails to satisfy the parabolic maximum principle, on each sufficiently small cylinder $W=V \times\left(t_{1}, t_{0}\right) \in \mathscr{B}_{p}(U)$ having $x_{0} \in V$ and $t_{1}<t_{0}$. That is, condition (a) in Theorem 4.1 fails.

ACKNOWLEDGments. Laugesen thanks the Department of Mathematics and Statistics at the University of Canterbury for hosting him during this work. And we are grateful to Norm Levenberg for asking stimulating questions, and to Juan Manfredi for useful references.

\section{REFERENCES}

1. Armitage, D. H., and Gardiner, S. J., Classical Potential Theory, London, Springer-Verlag, 2001.

2. Brelot, M., Lectures on Potential Theory, Second edition, Bombay, Tata Institute of Fundamental Research, 1967.

3. Crandall, M. G., Evans, L. C., and Gariepy, R. F., Optimal Lipschitz extensions and the infinity Laplacian, Calc. Var. Partial Differential Equations 13 (2001), 123-139.

4. Crandall, M. G., and Wang, P.-Y., Another way to say caloric, J. Evol. Equ. 3 (2003), 653-672. 
5. Crandall, M. G., and Zhang, J., Another way to say harmonic, Trans. Amer. Math. Soc. 355 (2003), 241-263.

6. Hervé, R.-M., Recherches axiomatiques sur la théorie des fonctions surharmoniques et du potentiel, Ann. Inst. Fourier (Grenoble) 12 (1962), 415-571.

7. Juutinen, P., Lindqvist, P., and Manfredi, J. J., On the equivalence of viscosity solutions and weak solutions for a quasilinear equation, SIAM J. Math. Anal. 33 (2001), 699-717.

8. Littman, W., Generalized subharmonic functions: monotonic approximations and an improved maximum principle, Ann. Scuola Norm. Sup. Pisa Cl. Sci. (4) 17 (1963), 207-222.

9. Miranda, C., Partial Differential Equations of Elliptic Type, 2nd ed., Berlin, Springer-Verlag, 1970.

10. Ohnuma, S. and Sato, K., Singular degenerate parabolic equations with applications to the p-Laplace diffusion equation, Comm. Partial Differential Equations 22 (1997), 381-411.

11. Tautz, G., Zur Theorie der ersten Randwertaufgaben, Math. Nachr. 2 (1949), 279-303.

12. Watson, N. A., Generalizations of the spherical mean convexity theorem on subharmonic functions, Ann. Acad. Sci. Fenn. Ser. A I Math. 17 (1992), 241-255.

DEPARTMENT OF MATHEMATICS

UNIVERSITY OF ILLINOIS

URBANA, IL 61801

USA

E-mail: Laugesen@math.uiuc.edu
DEPARTMENT OF MATHEMATICS AND STATISTICS UNIVERSITY OF CANTERBURY

PRIVATE BAG 4800

CHRISTCHURCH

NEW ZEALAND

E-mail: N.Watson@math.canterbury.ac.nz 